\title{
Reversal of TREM-1 ectodomain shedding and improved bacterial clearance by intranasal metalloproteinase inhibitors
}

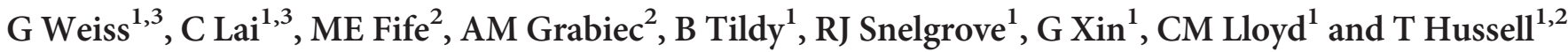

Triggering receptor expressed on myeloid cells-1 (TREM-1) is expressed on neutrophils and monocyte/macrophages and amplifies Toll-like receptor-mediated inflammation during infection. TREM-1 also exists in an antagonistic soluble form (sTREM-1) that has been used as a peripheral biomarker in sepsis, though the mechanisms of its release are not entirely clear. The requirement of TREM-1 in single microbial infections is controversial, with some studies showing a protective role and others a contribution to immunopathology. Furthermore, the role of membrane-bound and sTREM-1 in polygenic infections is currently unknown. In a mouse co-infection model where preceding viral infection greatly enhances bacteria co-infection, we now determine a mechanisms for the striking increase in sTREM-1 and the loss of TREM-1 on surface of neutrophils. We identified a matrix metalloproteinase (MMP)- 9 cleavage site in TREM-1 and that the increase of MMP-9 in bronchoalveolar lavage fluid mirrors STREM-1 release. In vitro studies with neutrophils and MMP-9 and the reduction of STREM-1 in vivo after MMP-9 inhibition verifies that this enzyme cleaves TREM-1. Intriguingly, MMP-9 inhibition significantly reduces bacterial load and ensuing immunopathology in a co-infection model. This highlights MMP-9 inhibition as a potential therapeutic via blocking cleavage of TREM-1.

\section{INTRODUCTION}

Bacterial super-infections often follow pulmonary virus infection and are a major cause of excess morbidity and mortality. ${ }^{1}$ Enhanced disease is due to a combination of excessive host immune responses and an exponential increase in bacterial replication that invades the lung and disseminates to distant organs. ${ }^{2}$ Membrane-bound pathogen recognition receptors on innate immune cells respond either alone or in combination to allow diversification of the early immune response. Co-operation between receptors draws in a variety of signaling cascades that shape an appropriate response, or in the case of some co-receptors, timely termination of inflammation. The triggering receptors expressed on myeloid cells (TREM) family either amplifies (TREM-1) or dampens (TREM-2) inflammation. ${ }^{3}$ TREM-1 amplifies the inflammatory response to Toll-like receptor signaling by recruiting the transmembrane adapter molecule DAP12, which recruits the tyrosine kinase Syk and leads to the activation of PI3K and MAPK. ${ }^{4}$ TREM- 1 signaling also enhances NF- $\kappa \mathrm{B}$ induced cytokine production in dendritic cells via caspase-recruitment domain protein (CARD) $-9^{5}$ and downregulates suppressor of cytokine signaling-1 (SOCS-1) by inducing the expression of miR-155 in macrophages and lungs of mice treated with LPS. ${ }^{6}$

Like many membrane bound receptors, TREM- 1 also exists in soluble form (sTREM-1). Although the function of sTREM-1 is unknown, it is likely to negatively regulate membrane TREM1 receptor signaling through competition with the ligand(s). sTREM-1 is under investigation as a biomarker in a variety of infectious and non-infectious inflammatory conditions and is detected in the bronchoalveolar lavage (BAL) fluid in pneumonia $^{7}$ and in the serum ${ }^{8,9}$ and urine ${ }^{10}$ of septic patients. It is a marker of neonatal sepsis ${ }^{11}$ and predicts shock and death. ${ }^{12}$ sTREM-1 and its gene polymorphisms are also associated with sepsis prognosis ${ }^{13}$ and high sTREM-1 is predictive of Systemic Inflammatory Response Syndrome $(\text { SIRS })^{14}$ after surgery. TREM-1 and its soluble form are

\footnotetext{
${ }^{1}$ National Heart and Lung Institute, Department of Inflammation, Development \& Repair, Imperial College London, London, UK and ${ }^{2}$ Manchester Collaborative Centre for Inflammation Research (MCCIR), Manchester, UK. Correspondence: T Hussell (tracy.hussell@manchester.ac.uk)

${ }^{3}$ These authors contributed equally to this work. 
therefore potential diagnostic markers for infectious and chronic inflammatory diseases and also potential future targets for therapeutic manipulation. ${ }^{15,16}$ In order for STREM- 1 to be a reliable marker of disease, a greater understanding of the cells relevant to its release and mechanisms of its cleavage are required. Furthermore, the long term impact of reducing TREM-1 cleavage into the soluble form is currently unknown.

Secondary bacterial infections are a consequence of many lung inflammatory diseases and arise due to defective microbicidal responses in the remodeled airspaces. ${ }^{17} \mathrm{We}$ reasoned that the release of sTREM-1 is at least partly responsible for impaired innate immune responses to bacteria following influenza virus infection. We now report a matrix metalloproteinase (MMP)-9 cleavage site within the TREM-1 sequence and demonstrate an in vivo correlation between MMP-9 expression, the appearance of sTREM-1 in airway lavage and neutrophil recruitment during pulmonary influenza infection. This correlation is amplified in influenza virus/ bacterial super-infected mice. Inhibition of MMP-2/9 decreases sTREM- 1 and restores membrane-bound TREM-1 and antibacterial immunity that prevents mortality following bacterial superinfection. Although cleavage of immune stimulatory receptors such as TREM-1 is an efficient means to curtail inflammatory responses, it may have deleterious effects if the pathogen has not been completely cleared. MMP-9 is raised in a variety of pathologies associated with infectious complications. We therefore conclude that inhibition of MMP-9 and TREM-1 cleavage represents novel therapeutic targets for the alleviation of secondary bacterial pneumonia.

\section{RESULTS}

\section{Influenza infection increases susceptibility to Streptococcus pneumoniae superinfection}

The murine model of bacterial superinfection following influenza virus is well established with high reproducibility. ${ }^{18}$ Similar to prior studies, we show that uninfected mice gain weight whereas intranasal influenza PR8 (H1N1) virus infection causes substantial weight loss, peaking on day 7 after which they begin to recover (Figure 1a). Phosphatebuffered saline (PBS) mock-infected mice administered $10^{6}$ colony-forming unit (CFU) S. pneumoniae (Strep) intranasally on day 7 did not lose weight and were similar to PBS alone controls. However, intranasal infection with S. pneumoniae 7 days after influenza infection (PR8 + Strep) caused extensive weight loss and the mice had to be killed. Co-infection was accompanied by enhanced total cells in the BAL (Figure 1b) and lung (Figure 1c), enhanced neutrophilia (Figure 1d), elevated levels of tumor necrosis factor (TNF)- $\alpha$ (Figure 1e) and exponentially higher bacterial loads in the BAL (Figure 1f), lung (Figure 1g) and blood (Figure 1h) of co-infected mice.

\section{Excessive release of MMP-9 and STREM-1 during secondary bacterial superinfection}

We next determined the expression of sTREM-1, TREM- 1 and MMP-9 in our murine model of bacterial superinfection following influenza virus. sTREM-1 levels in the BAL of PBS control mice were very low, significantly increased in mice infected with influenza alone and S. pneumoniae alone, but were strongly elevated in co-infected mice (Figure 2a). A similar pattern, though at lower concentration, was observed measuring sTREM-1 in the serum (Figure 2b). MMP-9 expression in the BAL followed the same pattern with an average 12- and 9-fold increase in co-infected mice compared with those infected with influenza or S. pneumoniae alone, respectively (Figure 2c). The correlation between MMP-9 and sTREM-1 by Spearman rank test was highly significant $(R=0.755, P<0.0001$; Figure 2d).

As it has been suggested that sTREM-1 is generated via cleavage of membrane-bound TREM-1 from the cell surface, ${ }^{19}$ and the co-infection model recruits a large number of neutrophils that express it, we analyzed whether there is a correlation between membrane-anchored TREM-1 on neutrophils and the release of sTREM-1 in the BAL. Both PBS control and influenza infected mice expressed high levels of TREM-1 on lung neutrophils ( $24 \%$ and $22 \%$, respectively) and displayed low levels of sTREM-1 in the BAL (Figure 2e). By contrast bacterial infection alone, and especially following influenza infection, reduced membrane-bound TREM-1 on neutrophils and increased sTREM-1. This result was also obtained applying total neutrophil numbers (Figure 2f). Thus, it is indicated that the balance of membrane bound TREM-1 versus sTREM-1 is altered during inflammation of the lung, which may lead to inefficient clearance of bacteria, resulting in systemic dissemination and mortality.

\section{Trem-1 contributes to neutrophil degranulation}

Neutrophils constitute the main cellular defence against S. pneumoniae. ${ }^{20}$ At the site of infection they phagocytose bacteria that are killed by reactive oxygen species ${ }^{21,22}$ and serine proteases cathepsin G, elastase and proteinase 3 released from intracellular granules. ${ }^{23}$ Neutrophils also express patternrecognition receptors to sense and respond to pathogens. ${ }^{24}$ The function of TREM-1, and the impact of sTREM-1, however, is currently unknown. We therefore purified neutrophils from bone marrow and cultured them with LPS in the presence of a TREM-1 agonist antibody or an antagonistic TREM-1 peptide (LP17). Agonism of TREM-1 had no effect on phagocytosis of bacterial particles and ROS production (data not shown), but enhanced degranulation, which is reflected by the upregulation of CD63 (Figure 3a), and CD11b expression (Figure 3b) whereas LP17 had the opposite effect (Figure 3c,d). Thus TREM-1 potentiates certain aspects of neutrophil anti-bacterial immunity.

Inhibition of MMP-2/9 prevents bacterial infiltration and the proinflammatory response during secondary bacterial superinfection

The TREM-1 sequence (MRKAGLWGLL CVFFVSEVKA AIVLEEERTD LVEQTLTVKCPFNIMKYAN SQKAWQRLPD GKEPLTLVVT QRPFTRPSEV HMGKFTLKHD PSEAMLQVQM TDLQVTDSGL YRCVIYHPPN DPVVLFHPVR LVVTKGSSDVFTPVIIPITR ITERPILITT KYSPSDTTTT 

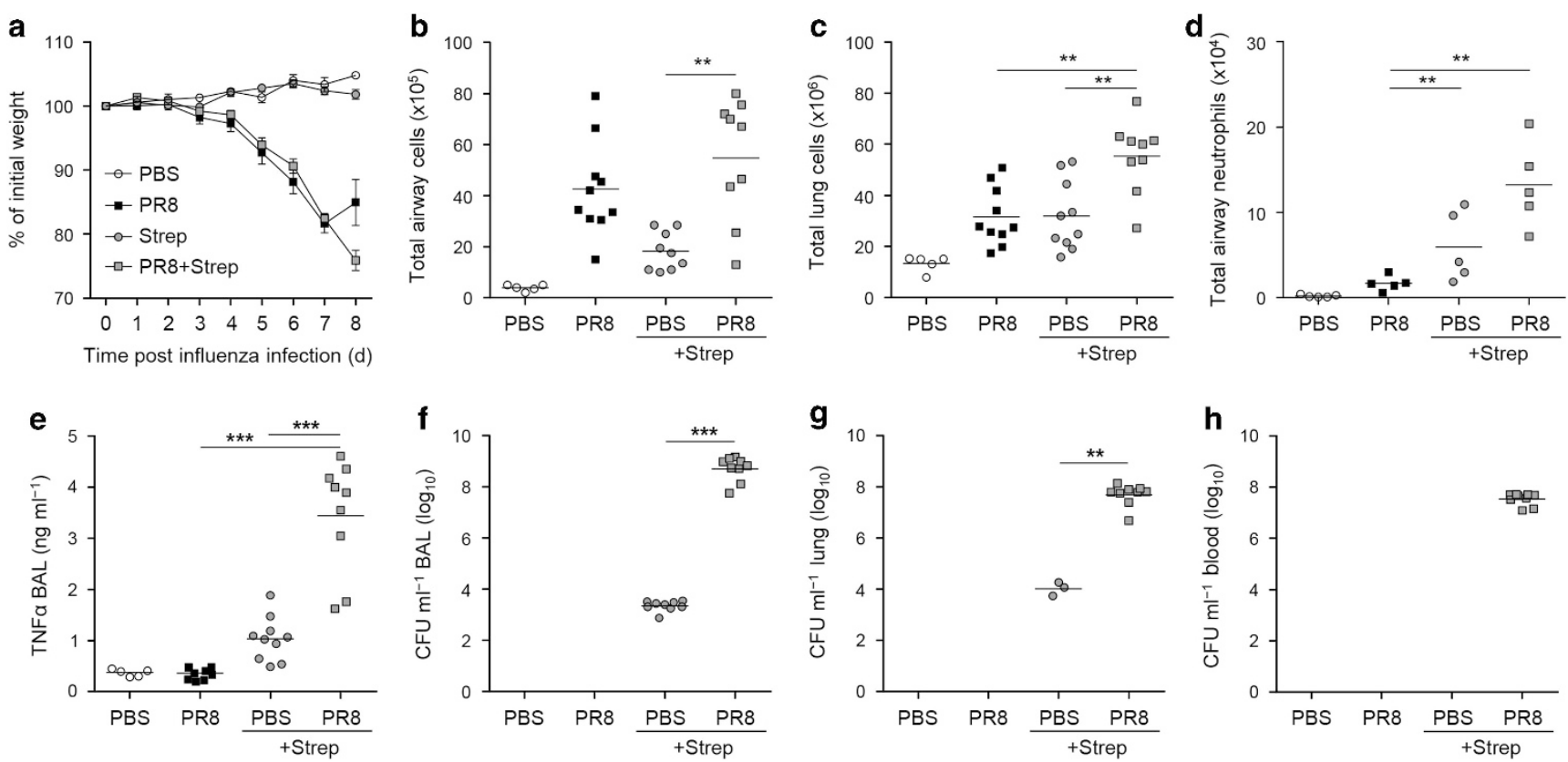

Figure 1 Influenza infection increases susceptibility to Streptococcus pneumoniae superinfection. BALB/c mice were infected with 7 plaque-forming units (PFU) of PR8 influenza A virus, followed by $10^{6} \mathrm{CFU}$ S. pneumoniae on day 7 and analyzed $24 \mathrm{~h}$ post-S. pneumoniae infection (PR8 + Strep). Phosphate-buffered saline (PBS) mice, mice infected with influenza alone (PR8) and S. pneumoniae (Strep) alone were used as a control. Body weight was measured daily and is expressed as percentage of original body mass (a). Total live cells in bronchoalveolar lavage (BAL) (b) and lung (c) was determined by trypan blue exclusion. Neutrophils were identified by flow cytometry (d). Airway levels of tumor necrosis factor (TNF)- $\alpha$ (e) was determined by enzyme-linked immunosorbent assay (ELISA). Viable bacterial colony-forming units (CFU) recovered from the BAL (f), lung tissue (g) and peripheral blood (h) were enumerated by counting colonies on agar. Data are expressed as mean \pm s.e.m., $n=6-10$ mice per group from two independent experiments. Statistical significance was determined by Mann-Whitney $U$-test between relevant groups, ${ }^{* \star} P<0.01,{ }^{* * *} P<0.001$.

\section{RSLPKPTAVV SSPGLGVTII NGTDADSVST SSVTISVICG} IISKSLVFII LFIVTKRTFG) contains an MMP-9 cleavage site (underlined). The possible sequence for an MMP-9 cleavage site is $\mathrm{P}-\mathrm{X}-\mathrm{X}-\mathrm{H}-\mathrm{T}$, where $\mathrm{X}$ is any amino acid and $\mathrm{H}$ is hydrophobic.

To formally prove that MMP-9 causes the cleavage of membrane-bound TREM-1, we incubated murine bonemarrow-derived neutrophils with unactivated MMP-9 or AMPA activated MMP-9 (as described by Marcy AL et $a l^{25}$ ). Stimulation of bone-marrow-derived neutrophils with activated MMP-9 reduced the expression of membrane-bound TREM-1 and increased the levels of sTREM-1 compared with unactivated MMP-9 (Figure 4a).

We next infected mice intranasally (i.n.) with influenza virus strain PR8 and with S. pneumoniae on day 7. One co-infected group was treated with an MMP-2/9 inhibitor i.n. on day 6 and day 7 , whereas the other group received vehicle only. Mice were sacrificed on day 8 (Figure $4 \mathbf{b}$ ). The activation status of MMP-9 was assessed by zymography in the BAL of influenza and S. pneumoniae infected mice administered the MMP-2/9 inhibitor and compared with co-infected mice that received vehicle only (Figure 4c). Co-infected mice showed strong activity of MMP-9, which was substantially reduced upon inhibitor treatment. Levels of sTREM-1 in BAL (Figure 4d) and serum (Figure 4e) and MMP-9 protein levels in BAL (Figure 4f) and serum (Figure $\mathbf{4 g}$ ) were significantly suppressed in the inhibitor treated group. The activity (data not shown) and expression (Figure $4 \mathbf{h}$ ) of MMP-2 in the BAL was not affected by the MMP-2/9 inhibitor, though this is likely to reflect the very low levels found in this model compared with MMP-9 (data not shown). The correlation of sTREM-1 (Figure 4i) with CFU was significant by Spearman Rank test $(R=0.744$; $P=0.002$ ). However, similar to the correlation between CFU and MMP-9 levels (Figure 4j), a certain threshold of bacteria had to be present in order for sTREM-1 to increase. In both cases this threshold appeared to be above $10^{6} \mathrm{CFU}$ per $\mathrm{ml}$ (Figure $4 \mathbf{i}, \mathbf{j})$. Our results indicate that inhibition of MMP-2/9 reduces the release of TREM-1 in vivo.

\section{Inhibition of MMP-2/9 increases the expression of TREM-1 on neutrophils in the lung during secondary bacterial superinfection}

A decrease in sTREM- 1 could reflect a reduction in recruited neutrophils rather than a decrease in sTREM-1 release. During infection neutrophils are the main cellular source of MMP-9. ${ }^{26}$ As one role of MMP-9 is to cleave IL- $8,{ }^{27}$ (mouse homolog $\mathrm{KC}),{ }^{28}$ to form a more potent neutrophil chemoattractant we next determined the impact of MMP-2/9 inhibition on cell recruitment. Both vehicle and MMP-2/9 inhibitor treatment of co-infected mice displayed equivalent airway (Figure 5a) and only partially reduced lung (Figure 5b) cellularity. Importantly, expression of TREM-1 on neutrophils was significantly higher in BAL (Figure 5c,d) and lung (Figure 5e,f) of mice treated with the MMP-2/9 inhibitor compared with the control group. 

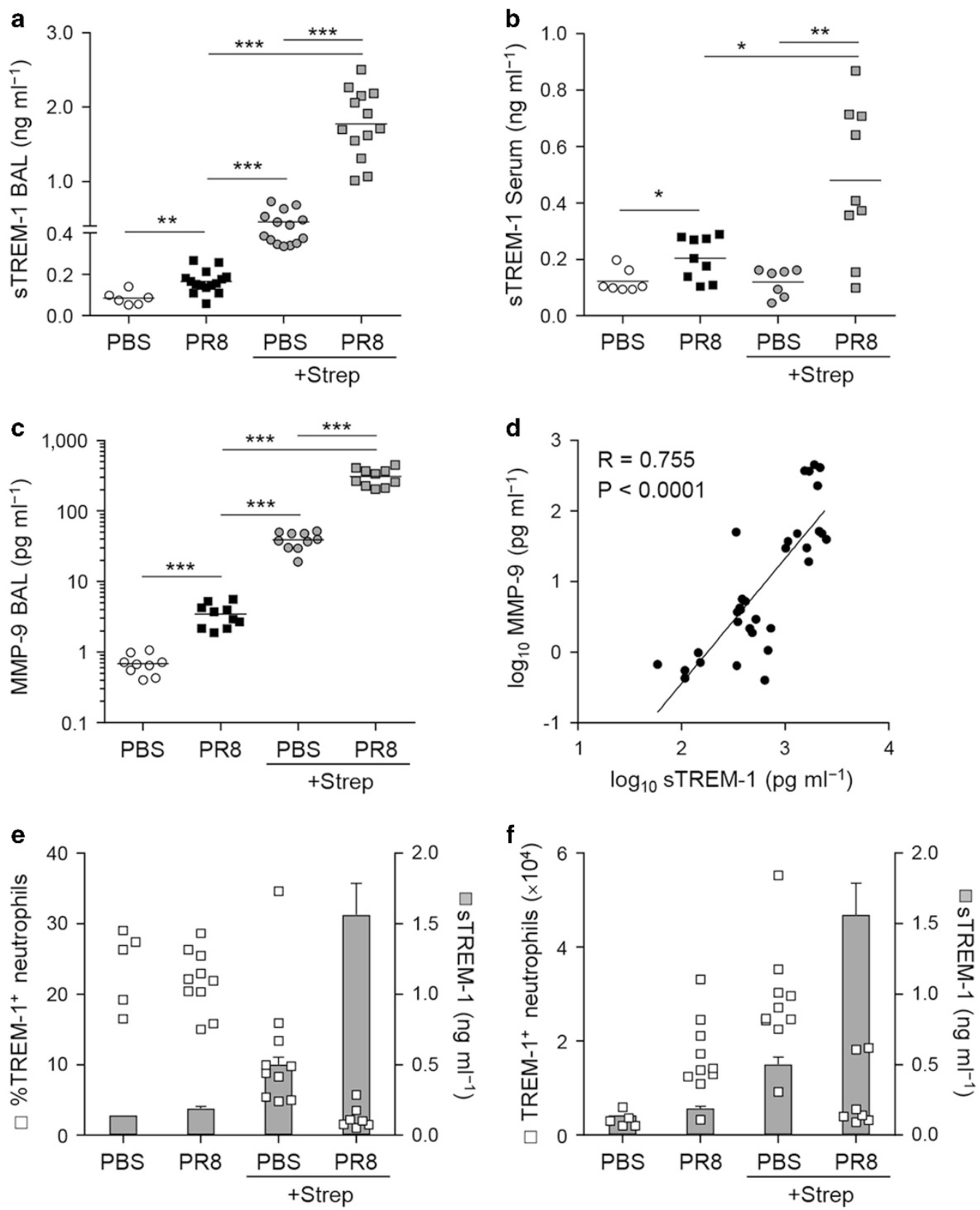

Figure 2 Excessive release of matrix metalloproteinase (MMP)-9 and soluble triggering receptor expressed on myeloid cells-1 (sTREM-1) during secondary bacterial superinfection. BALB/c mice were infected with 7 plaque-forming units (PFU) of PR8 influenza A virus, followed by $10^{6}$ PFU $S$. pneumoniae on day 7 and analyzed $24 \mathrm{~h}$ post-S. pneumoniae infection (PR8 + Strep). Control groups as per Figure 1. (a) Expression of soluble triggering receptor expressed on myeloid cells-1 (sTREM-1) in bronchoalveolar lavage (BAL) and (b) serum and (c) Matrix metalloproteinase (MMP)-9 in BAL determined by enzyme-linked immunosorbent assay (ELISA). Spearman Rank correlation between BAL MMP-9 and sTREM (d, each point represents an individual mouse). STREM-1 (gray bars) measured by ELISA compared with (e) percentage of neutrophils or (f) total neutrophils expressing TREM-1 ${ }^{+}$ $(\square)$ in the lung measured by FCM. Naive, uninfected mice were analyzed as a control (phosphate-buffered saline (PBS)). Data are expressed as mean \pm s.e.m., $n=6-10$ mice from two independent experiments. Statistical significance was determined by Mann-Whitney $U$-test between relevant groups, ${ }^{*} P<0.05,{ }^{* *} P<0.01,{ }^{* \star *} P<0.001$.

Administration of the MMP-2/9 inhibitor increased the expression of TREM-1 on neutrophils in the BAL 4.3-fold and in the lung 4.5-fold. A similar result was obtained for total numbers of lung neutrophils where membrane-bound TREM-1 was restored to pre-infection levels (Figure 5f). Comparison of the release of sTREM-1 with membrane- bound TREM-1 revealed that high amounts of sTREM-1 correlated with low expression of TREM-1 on neutrophils in BAL (Figure 5g) and lung (Figure 5h). Thus, MMP-9 contributes to bacterial super-infection by cleaving membrane-bound TREM-1, preventing the timely removal of the bacterium from the influenza infected lung. 

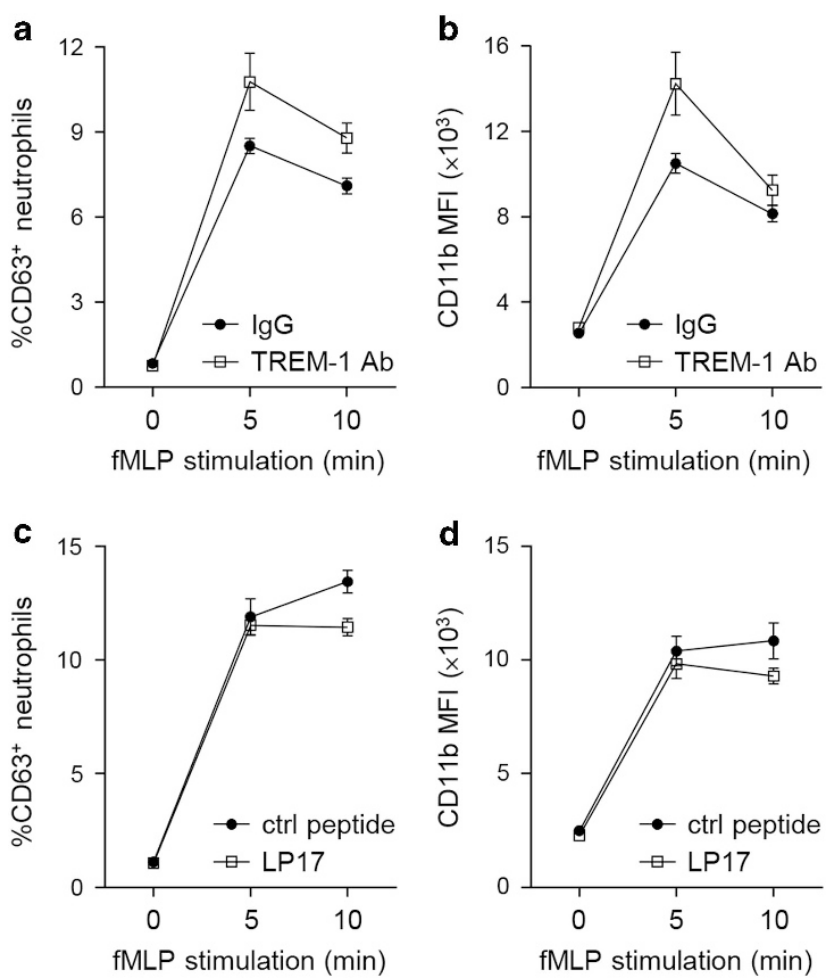

Figure 3 Triggering receptor expressed on myeloid cells-1 (TREM-1) regulates neutrophil degranulation and integrin expression. Flow cytometric analysis of cell surface CD63 (LAMP3) $(\mathbf{a}, \mathbf{c})$ and CD11b (b,d) on bone marrow neutrophils pre-incubated with $10 \mu \mathrm{g} \mathrm{ml}^{-1}$ control goat IgG or $10 \mu \mathrm{g} \mathrm{ml}^{-1}$ activating TREM-1 antibody (a,b) and $1 \mu \mathrm{g} \mathrm{ml}^{-1}$ control peptide or $1 \mu \mathrm{g} \mathrm{ml}^{-1} \mathrm{LP} 17$ peptide (c,d), followed by stimulation with $10 \mathrm{~nm}$ fMLP for up to $15 \mathrm{~min}$. Data are presented as the mean \pm s.e.m. of four mice per group.

\section{Inhibition of MMP-2/9 reduces the expression of MMP-9 and sTREM-1 during secondary bacterial superinfection}

As our results show that secondary bacterial superinfection following influenza results in excessive release of MMP-9 and sTREM-1, we next addressed whether inhibition of MMP-9 in vivo has an impact on disease outcome. As our results show that secondary bacterial superinfection following influenza results in excessive release of MMP-9 and sTREM-1, we next addressed whether inhibition of MMP-9 in vivo has an impact on disease outcome using the MMP-2/9 inhibitor co-infection model infection model described in Figure $\mathbf{4 b}$. No difference in weight loss was observed in the inhibitor treated mice compared with the PR8 influenza and S. pneumoniae infected control mice administered vehicle only (Figure 6a). As demonstrated previously, infection of mice with $S$. pneumoniae 7 days post influenza leads to a high bacterial load in the airway, lung and blood (Figure 1f-h). However, treatment with the MMP-2/ 9 inhibitor significantly reduced bacterial titers in the BAL (Figure 6b) and lung (Figure 6c) and, remarkably, completely prevented sepsis (Figure $\mathbf{6 d}$ ). In contrast to our co-infected control group displaying a high bacterial load $\left(9 \times 10^{7} \mathrm{CFU}\right.$ per $\mathrm{ml})$, no bacteria were detected in the blood of mice treated with the inhibitor. We also observed a significant reduction in the airway proinflammatory cytokines interleukin (IL)-6
(Figure 6e), IL-1 $\alpha$ (Figure 6f) and TNF- $\alpha$ (Figure 6g) compared with the control group, whereas the expression of interferon- $\gamma$ remained unchanged (Figure 6h). Noteworthy, although secondary bacterial infection leads to a systemic disease, these results were achieved via intranasal administration of the inhibitor only. Altogether, our data indicate that inhibition of MMP-2/9 strongly reduces the bacterial load in $\mathrm{BAL}$ and lung, prevents sepsis and the proinflammatory response.

\section{DISCUSSION}

The resolution of inflammation requires integrated responses that return damaged tissues to homeostasis. The release of soluble ectodomains of immune stimulatory receptors by proteases, which are upregulated during inflammation, is an effective method of producing anti-inflammatory antagonists (as proposed for sTREM-1 (ref. 20)). A classic example is the release of soluble TNF receptor trimers that sequester TNF- $\alpha$ preventing activation of membrane-bound receptors. Other examples include shed L-selectin that binds PSGL1 on endothelial cells curtailing cell adhesion ${ }^{21}$ and JAM-A that binds LFA-1 on leukocytes impeding transmigration. ${ }^{22}$ MMP-9 is released during a variety of infections, which we now show is elevated further upon bacterial super-infection. This additional production likely reflects the recruitment of large number of neutrophils containing MMP-9 in pre-formed granules. ${ }^{23,24}$ MMP-9 promotes endothelial permeability, assists inflammatory cell migration through the breakdown of vascular basement membranes and extracellular matrix ${ }^{29}$ and is associated with the release of membrane-anchored signaling molecules in response to different stimuli. For example, MMP9 activates the inactive form of TGF- $\beta$ and IL- $1 \beta^{30}$ via cleavage. Some cleaved ectodomains are inert or have advantageous biological activity. ${ }^{31}$ Others may, however, contribute to the pathological process. During chronic Staphylococcus aureus lung infection the activation of ADAM10 cleaves epidermal growth factor stimulating mucus production that contributes to airway obstruction. Genome-wide association studies implicate ADAM33 in asthma and airway hyper-reactivity and TGF- $\beta$ causes ectodomain shedding of ADAM33 that contributes to lung remodeling. Furthermore, MMP-7 increases ectodomain shedding of E-cadherin from epithelia, leading to loss of epithelial coherence. $^{32}$

We now show TREM-1 cleavage by MMP-9, similar to that reported for peritoneal and RAW macrophages in vitro, ${ }^{33}$ and that MMP-9 inhibition restores neutrophil membrane TREM1 expression. Interestingly, MMP-9 also cleaves surfactant protein $\mathrm{D}$ decreasing its ability to aggregate bacteria and facilitate phagocytosis by alveolar macrophages. ${ }^{34}$ This suggests that MMP-9 inhibition would restore a number of neutrophil anti-bacterial mechanisms. Definitive proof of a direct effect of MMP-9 on TREM-1 cleavage is difficult to obtain however, and would require generating a knockin of TREM-1 expressing a mutated MMP-9 cleavage site. Although in vivo MMP-2/9 neutralisation would suggest other mechanisms are not involved with TREM-1 cleavage, we cannot rule out a role 

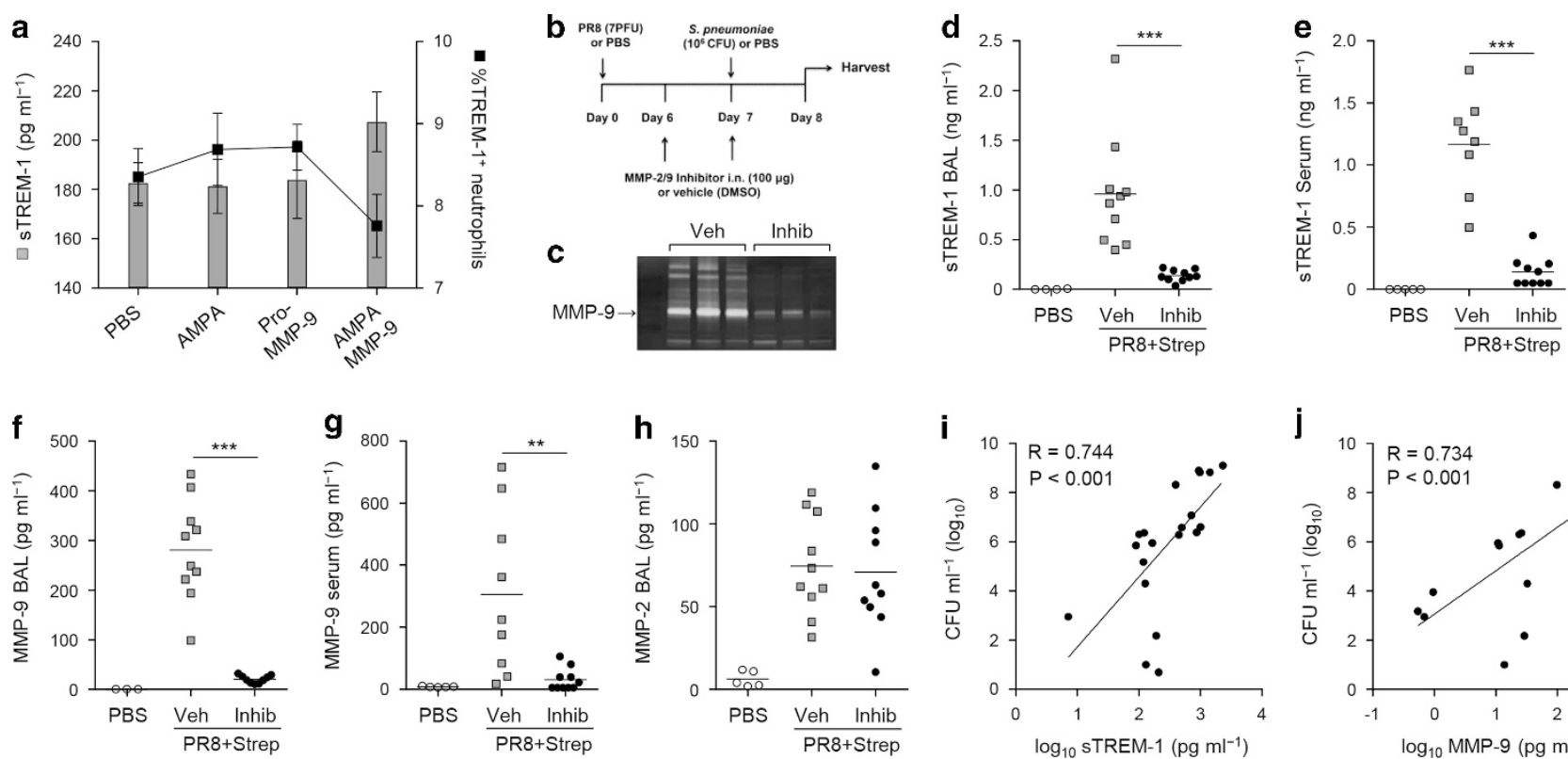

Figure 4 Inhibition of matrix metalloproteinase (MMP)-2/9 reduces the expression of MMP-9 and soluble triggering receptor expressed on myeloid cells-1 (sTREM-1) during secondary bacterial superinfection. Murine bone-marrow-derived neutrophils incubated with $p$-aminophenylmercuric acetate (APMA), unactivated (Pro) MMP-9 and APMA-activated MMP-9. sTREM-1 expression measured by enzyme-linked immunosorbent assay (ELISA) in cell culture supernatants (bars) and expression of TREM-1 on neutrophils determined by FACS (line) (a). Data are from three independent experiments. BALB/c mice were infected with 7 PFU of PR8 influenza A virus, followed by $10^{6} \mathrm{CFU}$ S. pneumoniae on day 7 and analyzed $24 \mathrm{~h}$ post-S. pneumoniae infection. On day 6 and on the day of $S$. pneumoniae infection an MMP-2/9 inhibitor was administered i.n. (100 $\mu$ g) or vehicle alone (b). Gelantine zymogram of MMP-9 activity (c). Expression of sTREM-1 in bronchoalveolar lavage (BAL) (d), serum (e), MMP-9 in BAL (f) and serum (g) and MMP-2 in BAL (h) determined by enzyme-linked immunosorbent assay (ELISA). Spearman Rank correlation between CFU and sTREM-1 (i) and MMP-9 (j). Data are expressed as mean \pm s.e.m., $n=6-10$ mice per group from two independent experiments. Statistical significance was determined by Mann-Whitney $U$-test between relevant groups, ${ }^{* \star} P<0.01,{ }^{\star \star \star} P<0.001$.
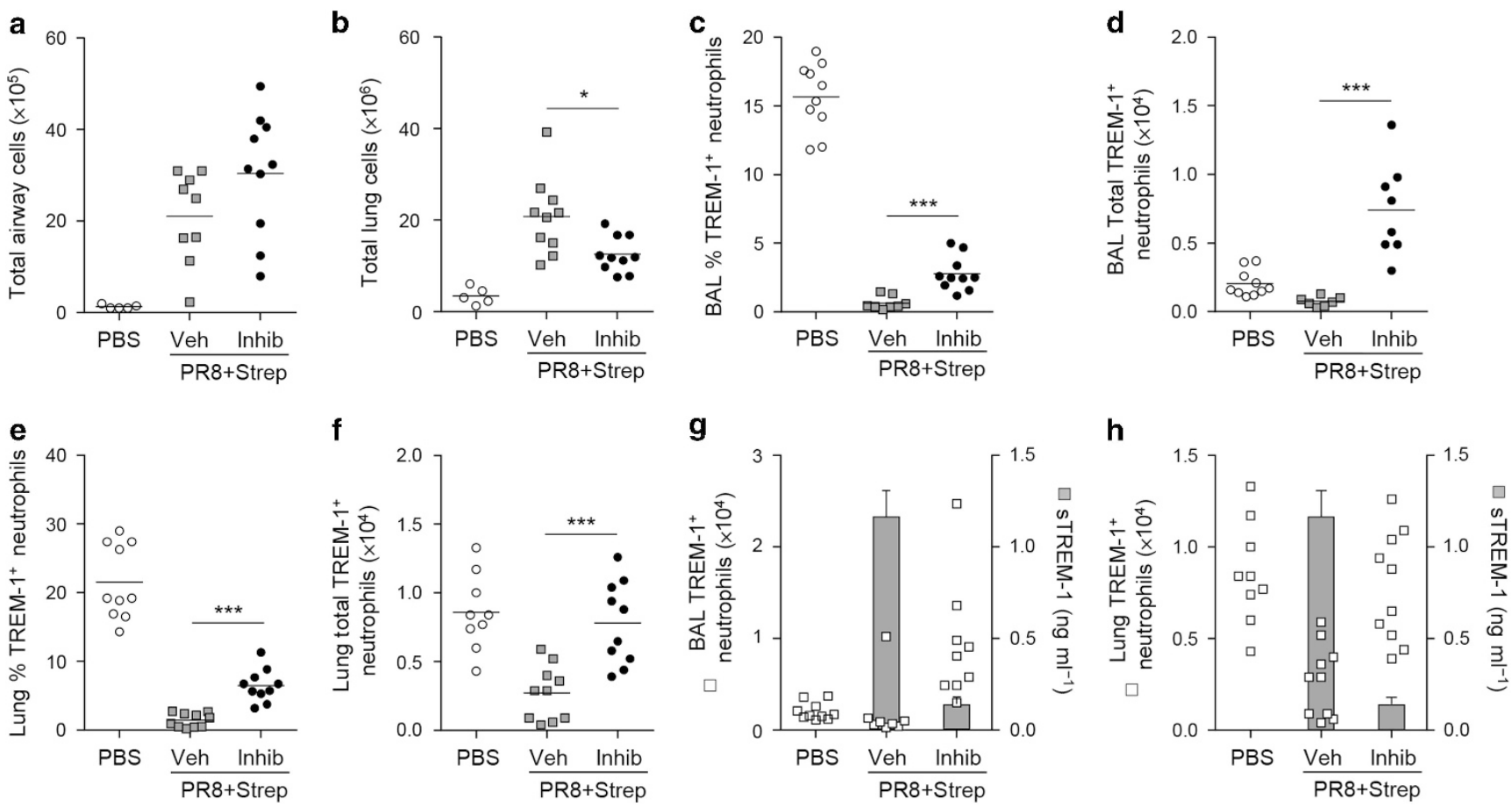

Figure 5 Inhibition of matrix metalloproteinase (MMP)-2/9 increases the expression of triggering receptor expressed on myeloid cells-1 (TREM-1) on neutrophils in the lung during secondary bacterial superinfection. BALB/c mice were treated according to Figure $\mathbf{4 b}$. Total live cells in bronchoalveolar lavage (BAL) (a) and lung (b) was determined by trypan blue exclusion. Percentage (c) and total numbers (d) of TREM-1 expressing neutrophils in BAL and percentage (e) and total numbers (f) of TREM-1 expressing neutrophils in the lung was determined by FCM. Correlation between soluble TREM-1 (sTREM-1; gray bars) measured by enzyme-linked immunosorbent assay (ELISA) compared with total neutrophils ( $\square$ ) in the BAL (g) and lung (h). Data are expressed as mean \pm s.e.m., $n=6-10$ mice per group from two independent experiments. Statistical significance was determined by Mann-Whitney U-test between relevant groups, ${ }^{*} P<0.05,{ }^{* * *} P<0.001$. 

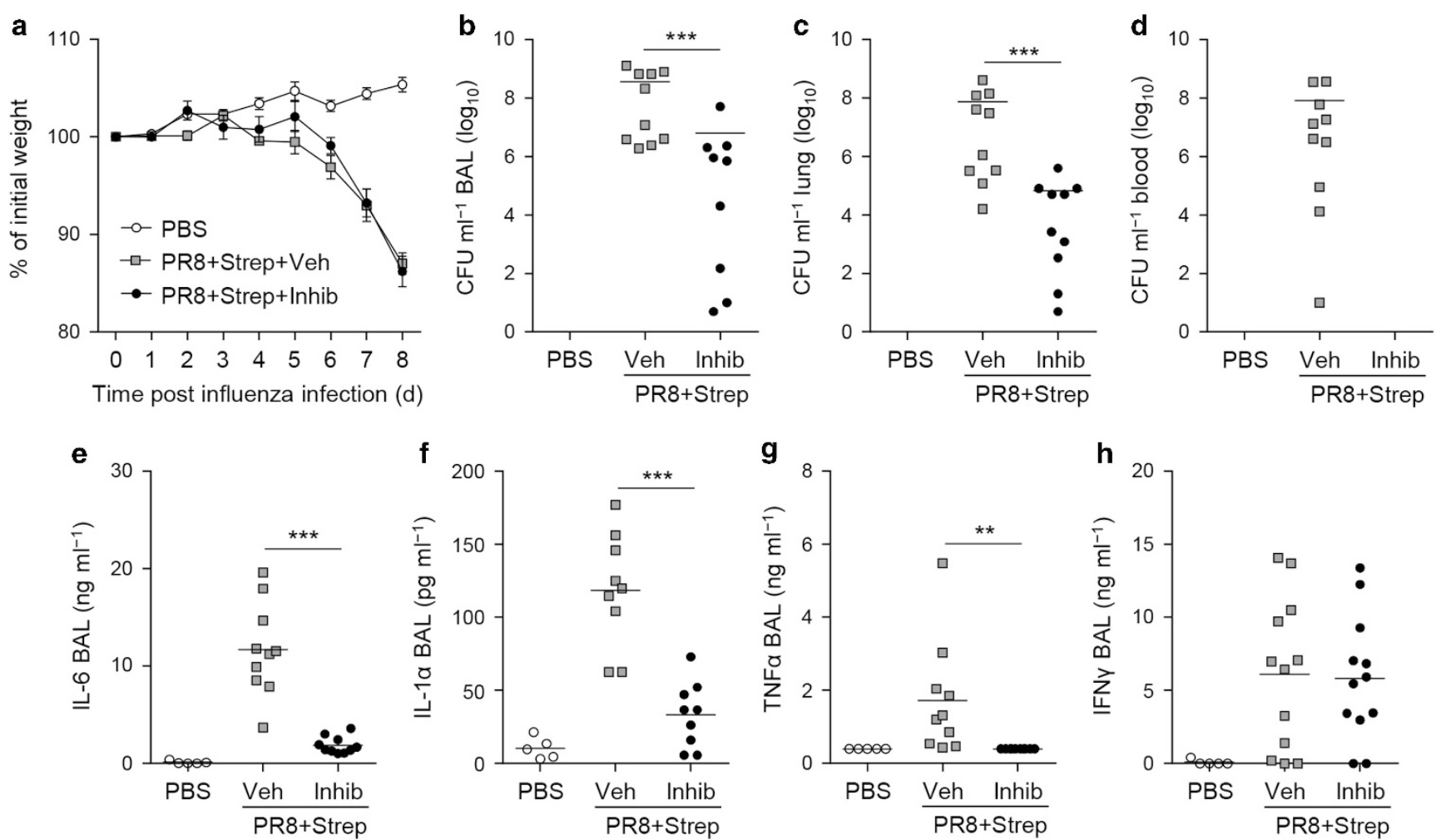

Figure 6 Inhibition of matrix metalloproteinase (MMP)-2/9 prevents bacterial infiltration and the proinflammatory response during secondary bacterial superinfection. BALB/c mice were treated according to Figure $4 \mathbf{b}$. Body weight was measured daily and is expressed as percentage of original body mass (a). Viable bacterial colony-forming units (CFU) recovered from the BAL (b), lung tissue (c) and peripheral blood (d) was enumerated on agar. Airway levels of interleukin (IL)- 6 (e), IL-1 $\alpha$ (f), tumor necrosis factor (TNF)- $\alpha(\mathbf{g})$ and interferon (IFN)- $\gamma$ (h) were enumerated by enzyme-linked immunosorbent assay (ELISA). Data are expressed as individual mice and mean \pm s.e.m., $n=6-10$ mice per group from two independent experiments. Statistical significance was determined by Mann-Whitney $U$-test between relevant groups, ${ }^{*} P<0.05$, ${ }^{\star * \star} P<0.001$.

for other proteases. Compelling associations combined with in vivo and in vitro studies however indicate a classic feedback loop curtailing neutrophil function that has deleterious effects on subsequent bacterial control.

Although cleavage of activation receptor ectodomains affords resolution, the timing of this cleavage is crucial, since early release will allow remaining pathogens to escape and ultimately lead to prolonged and exaggerated inflammation. Indeed, blockage of TREM-1 signaling causes severe proinflammatory responses to Streptococcus suis, whereas activation has the opposite effect. ${ }^{35}$ Similarly agonistic TREM-1 antibodies facilitate early clearance of $S$. pneumoniae single infection. ${ }^{7}$ We now additionally show that TREM- 1 facilitates neutrophil degranulation and hence bactericidal activity. Inflammation can therefore arise in the presence and absence of membrane TREM-1. Intact membrane bound TREM-1 slows the resolution of inflammation, whereas once cleaved bacteria grow exponentially leading to more immune stimulation. The timing of membrane to secreted TREM-1 is therefore critical. Secondary pulmonary bacterial infections are a feature of a wide range of lung inflammatory disorders (including asthma, COPD, cystic fibrosis and IPF) that are also associated with increased MMP-9 production. The link to soluble TREM-1, however, has not previously been investigated. In general the outcome depends on whether TREM-1 is neutralized or the gene deleted. Neutralization of TREM-1 attenuates inflammation, preventing experimentally induced bacterial septic shock ${ }^{36-42}$ and attenuating disease severity in mice infected with Leishmania major, influenza virus and Legionella pneumophila. ${ }^{43}$ However, the situation for Klebsiella pneumoniae appears to be different with TREM-1/3-deficient mice showing impaired host defence, ${ }^{44}$ similar to that observed for pneumococcal pneumonia ${ }^{45}$ and Pseudomonas aeruginosa ${ }^{8}$ in the same mice. These results are not necessarily contradictory since global knockout affects innate immunity from the outset, whereas therapeutic neutralisation does not.

Ectodomain cleavage of membrane-bound TREM-1 via MMP-9 during influenza virus infection associates with secondary bacterial super-infection that is lethal. Cleavage of TREM-1 is likely required to prevent influenza virusmediated damage, but has the disadvantage of creating a window of susceptibility to bacteria. Respiratory syncytial virus also stimulates MMP-9 release from human airway epithelium and is a biomarker of disease severity in mechanically ventilated children with respiratory syncytial virus lung infection. ${ }^{46}$ This suggests that common processes may be occurring in respiratory disease, which is important in the absence of knowing the etiological agent(s). We would propose that MMP-9 inhibition in those most severely affected by respiratory viral infection would restore membrane TREM-1 
expression and abolish the lethal synergistic viral-bacterial disease. However, additional effects of neutralizing these proteases would need to be taken into consideration.

Considering that MMP-2 and MMP-9 cooperate to cleave chemokines involved in neutrophil migration, ${ }^{47}$ particularly IL- $8{ }^{48}$ we were surprised to see little effect of these metalloproteinases on neutrophil numbers in the airways of bacterial super-infected influenza resolved mice. A partial reduction was, however, observed in lung tissue. A previous study of single influenza virus lung infection in MMP-9 knockout mice reports reduced neutrophil migration and disease pathogenesis. ${ }^{49} \mathrm{We}$ believe the mild effect on airway neutrophilia seen in our study reflects their over-whelming recruitment in this super-infection model that masks any subtle effect. It is also interesting that MMP-2/9 inhibition dramatically reduced the bacterial load, completely prevented sepsis and subsequently reduced many proinflammatory cytokines, except interferon- $\gamma$. This suggests beneficial effects on both bacterial clearance and immunemediated pathology. TNF and IL- 6 are dominant innate responses to bacterial infection whereas interferon- $\gamma$ is likely a remnant from the $\mathrm{T}$-cell response to the previous viral infection and less affected by MMP-2/9 inhibition. The restriction of bacteria from the blood of MMP-2/9 inhibitor treated mice may reflect reduced effects of metalloproteinases on endothelial permeability and the destruction of vascular basement membranes and extracellular matrix. ${ }^{21}$ Alternatively, our data of TREM-1 modulation of degranulation suggests that bacteria are cleared quickly without becoming invasive.

A TREM-1 genetic polymorphism rs2234246 has recently been described that correlates with susceptibility to septic shock in the Chinese Han population, ${ }^{50}$ though the implications of this for membrane bound and soluble TREM-1 are currently unclear. Soluble TREM-1 has also been utilized as a biomarker for disease severity, particularly in pneumonia and septic shock. Importantly, we show a relative insensitivity of serum soluble TREM-1 compared with airway lavage, which suggests that sputum may provide better differentiation of disease status. The timing of TREM-1 ectodomain shedding is clearly critical to the outcome of bacterial super-infections. In patients with underlying disease, this shedding may already have occurred by the time bacteria start to expand. Protease imbalance and elevated MMPs are a hallmark of an array of chronic diseases also susceptible to bacterial infection. Inhibition of MMP-2/9 is therefore an interesting and feasible target in order to redress the inability of innate immunity to respond rapidly.

\section{METHODS}

Pathogen stocks. Influenza A Puerto Rico/8/34 H1N1 (PR8), a kind gift from Prof Andreas Wack (National Institute for Medical Research, Mill Hill, UK), was stored at $-80^{\circ} \mathrm{C}$ in single-use aliquots. Wild type S. pneumoniae (serotype 2) strain D39 (NTCC 7466, National Collection of Type Cultures, London, UK) was a kind gift from Jeremy Brown (Centre for Respiratory Research, UCL, London, UK). Bacterial stocks were cultured at $37^{\circ} \mathrm{C}$ in $5 \% \mathrm{CO}_{2}$ in Todd-Hewitt broth (Sigma, Dorset, UK) supplemented with $0.5 \%$ yeast extract (OXOID, Basingstoke, UK) to an $\mathrm{OD}_{600}$ of $0.4\left(\sim 1 \times 10^{8}\right.$ colony-forming units (CFU)/ $\mathrm{mL}$ ) and stored at $-80{ }^{\circ} \mathrm{C}$ in $10 \%$ glycerol as single-use aliquots.
Secondary bacterial pneumonia model. Six- to 8-week old or 17-19g female BALB/c mice were purchased from Harlan Olac (Bicester, UK) and maintained in specific pathogen-free conditions at Bio Safety Level 2 in accordance with institutional and UK Home Office guidelines. Mice were anesthetized and i.n. inoculated with 15 plaque-forming units (PFU) influenza A virus PR8 strain in $50 \mu \mathrm{l}$ PBS or PBS alone as mock control. On day 7 post-viral infection, mice were inoculated i.n. with S. pneumoniae ( $\left.10^{6} \mathrm{CFU}\right)$. Disease severity and weight loss were monitored daily. Mice were culled $24 \mathrm{~h}$ post-bacterial infection by intraperitoneal injection of $3 \mathrm{mg}$ pentobarbitone (Animal Care, York, $\mathrm{UK})$ and exsanguination by cardiac puncture.

MMP-2/9 inhibitor administration during secondary bacterial pneumonia. Female $\mathrm{BALB} / \mathrm{c}$ mice were infected i.n. with PR8 influenza (7 PFU in $50 \mu \mathrm{lPBS}$ ) or PBS alone as control. On day 6 , mice were treated with MMP-2/9 inhibitor I (Calbiochem/Milipore, Hertfordshire UK,) i.n. (100 $\mu \mathrm{g}$ per mouse in $25 \mu \mathrm{l})$ in dimethylsulfoxide (DMSO). On day 7, mice were administered MMP-2/9 inhibitor i.n. ( $100 \mu \mathrm{g}$ per mouse in $25 \mu \mathrm{l})$ in DMSO or vehicle alone as control and infected i.n. with $S$. pneumoniae $\left(10^{6} \mathrm{CFU}\right.$ in $\left.25 \mu \mathrm{l} \mathrm{PBS}\right)$.

Sample recovery and cell isolation. Mice were sacrificed and blood, BAL fluid and lung tissue were harvested. For bacterial CFU analysis, $10 \mu \mathrm{l}$ of sodium heparin were added to $90 \mu \mathrm{l}$ of blood to prevent clotting (Leo Pharma, Berkshire, UK). The remaining blood was collected in serum-separating Microtainer tubes (BD Diagnostics, Oxford, UK) tubes, left to clot overnight at $4{ }^{\circ} \mathrm{C}$ prior to centrifugation at 8,000 r.p.m. for $5 \mathrm{~min}$ and stored at $-80^{\circ} \mathrm{C}$ for future analysis. BAL was performed by inflation of the lung three times with $0.4 \mathrm{ml}$ ice-cold PBS via an intratracheal cannula. Volume of $100 \mu \mathrm{l}$ were used for CFU analysis, the remaining BAL fluid was centrifuged at 1,200 r.p.m. for $5 \mathrm{~min}$ and the supernatant stored at $-80^{\circ} \mathrm{C}$. Lung tissue was chopped, incubated with 0.14 Wunsch units per ml Liberase (Roche, Welwyn Garden City, UK) and $50 \mu \mathrm{g} \mathrm{m}^{-1} \mathrm{DNAse}$ I (Roche) for $30 \mathrm{~min}$ at $37^{\circ} \mathrm{C}$ and passed through a $70 \mu \mathrm{M}$ cell strainer. Prior to centrifugation at 1,500 r.p.m. for $5 \mathrm{~min}, 100 \mu \mathrm{l}$ of lung cell suspension were used for CFU analysis. Red blood cells in BAL and lung cell suspensions were lysed with ammonium chloride potassium (ACK) buffer ( $0.15 \mathrm{M}$ ammonium chloride, $1 \mathrm{~m}$ potassium hydrogen carbonate and $0.01 \mathrm{~mm}$ EDTA, $\mathrm{pH}$ 7.2) for 3 mins and washed with RPMI-1640 medium (PAA, Buckinghamshire, UK) containing 10\% v/v foetal calf serum (FCS; Sigma). Cell viability was assessed by trypan blue exclusion.

Bacterial titres. Bacterial titers in BAL, lung and blood were determined by counting CFU from serial ten-fold dilutions of bacterial cell suspensions plated on Columbia blood agar plates supplemented with $5 \%$ defibrinated horse blood (TCS Bioscience, Buckingham, UK) and incubated for $24 \mathrm{~h}$ at $37^{\circ} \mathrm{C}$.

Neutrophil in vitro assay. Murine bone-marrow-derived neutrophils $\left(2 \times 10^{5}\right.$ cells) were stimulated with Pro-MMP-9 $\left(1 \mu \mathrm{g} \mathrm{ml}^{-1}\right.$; R\&D systems, Abingdon, UK), p-aminophenylmercuric acetate (APMA; $1 \mathrm{~mm}$, Sigma), and APMA-activated MMP-9 $\left(1 \mu \mathrm{g} \mathrm{ml}^{-1}\right)$ in RPMI1640 with $5 \%$ FCS serum at $37^{\circ} \mathrm{C}$ for $15 \mathrm{~min}$. Pro-MMP-9 was activated by pre-incubation with $1 \mathrm{~mm}$ APMA for $2 \mathrm{~h}$ at $37^{\circ} \mathrm{C}$. Expression of TREM-1 on the neutrophils was determined by FACS. Cell supernatants were collected and frozen at $-80^{\circ} \mathrm{C}$ prior to detection of sTREM-1 by enzyme-linked immunosorbent assay (ELISA).

In some experiments neutrophils were flushed out of bone marrow with ice-cold PBS and isolated using the neutrophil isolation kit (Miltenyi Biotec, Bisley, UK) as per the manufacturer instructions. Neutrophils $(100000)$ were incubated with $10 \mu \mathrm{g} \mathrm{ml}^{-1}$ anti-TREM-1 antibody, $10 \mu \mathrm{g} \mathrm{ml}^{-1}$ control Goat IgG (both from $\mathrm{R} \& \mathrm{D}$, Minneapolis, USA), $1 \mu \mathrm{g} \mathrm{ml}^{-1}$ LP17 (LQVTDSGLYRCVIYHPP) or $1 \mu \mathrm{g} \mathrm{ml}^{-1}$ LP17 control peptide (TDSRCVIGLYHPPLQVY) (both from Biotrend Chemical, Destin, USA) for $1 \mathrm{~h} .{ }^{51}$ Neutrophils were then stimulated with $10 \mathrm{~nm}$ fMLP (Sigma) 
for 5 and $10 \mathrm{~min}$, stained with anti-CD63 and anti-CD11b antibodies (both from eBioscience, Altrincham, UK) and analyzed by flow cytometry.

Flow cytometry. Cells isolated from BAL or lung tissue digest were stained with the live/dead marker Zombie Aqua (Biolegend, London, UK), Ly6G (eBioscience, Hatfield, UK) and TREM-1 (R\&D systems). Labeled cells were acquired on a BD Fortessa (BD Bioscience) and analyzed using FlowJo (Treestar, Ashland, OR, USA).

Mediator analysis. Murine sTREM-1, MMP-2 and MMP-9 were measured by Duoset ELISA kits (R\&D systems) and murine IL-6, IL$1 \alpha$, TNF- $\alpha$ and interferon- $\gamma$ (eBioscience) ELISA kits according to the manufacturer's instructions. MMP-9 activity was determined using Novex 10\% zymogram gelatin gels (Invitrogen, Paisley, UK). Images were captured and analyzed using myECL Imager software (ThermoScientific, Cheshire, UK).

Statistical analysis. Data were analyzed using Prism 5 (GraphPad Software, CA, USA). $P$-values were determined by the Mann-Whitney test comparing between 2 groups and are indicated on graphs as ${ }^{\star} P<0.05,{ }^{* *} P<0.01,{ }^{* * *} P<0.001$. Correlations were analyzed using Spearman's rank test.

\section{ACKNOWLEDGMENTS}

G.W. is a Marie Curie Research Fellow (303039). C.L. was funded by a PhD studentship from the M.R.C. \& Asthma UK Centre in Allergic Mechanisms of Asthma. R.J.S. is a Wellcome Trust Career Development Fellow (095707/Z/ 11/Z). T.H., A.M.G. and M.E.F. are supported by a pre-competitive, Open Innovation Centre grant from GlaxoSmithKline and AstraZeneca.

\section{AUTHOR CONTRIBUTIONS}

G.W. and C.L. generated all in vivo data presented. C.L. performed the neutrophil MMP-9 in vitro assay. M.E.F. and A.M.G. performed the studies relating to TREM-1 function on neutrophils. G.X. provided the proof of concept for the study. R.J.S and B.T. assessed MMP9 activity. T.H., G.W., C.M.L. and R.J.S. designed the research, generated the funds to perform the work and wrote the manuscript.

\section{DISCLOSURE}

The authors declare no conflict of interest.

Official journal of the Society for Mucosal Immunology

\section{REFERENCES}

1. Westermann, D., Savvatis, K., Schultheiss, H.P. \& Tschope, C. Immunomodulation and matrix metalloproteinases in viral myocarditis. J. Mol. Cell Cardiol. 48, 468-473 (2010).

2. Goulding, J., Godlee, A., Vekaria, S., Hilty, M., Snelgrove, R. \& Hussell, T. Lowering the threshold of lung innate immune cell activation alters susceptibility to secondary bacterial superinfection. J. Infect. Dis. 204, 1086-1094 (2011).

3. Ford, J.W. \& McVicar, D.W. TREM and TREM-like receptors in inflammation and disease. Curr. Opin. Immunol. 21, 38-46 (2009).

4. Tessarz, A.S., Weiler, S., Zanzinger, K., Angelisová, P., Horejsí, V. \& Cerwenka, A. Non-T cell activation linker (NTAL) negatively regulates TREM-1/DAP12-induced inflammatory cytokine production in myeloid cells. J. Immunol. 178, 1991-1999 (2007).

5. Hara, H. et al. The adaptor protein CARD9 is essential for the activation of myeloid cells through ITAM-associated and Toll-like receptors. Nat. Immunol. 8, 619-629 (2007).

6. Yuan, Z. et al. TREM-1-accentuated lung injury via miR-155 is inhibited by LP17 nanomedicine. Am. J. Physiol. Lung Cell Mol. Physiol. 310, L426L438 (2016).

7. Lagler, H. et al. TREM-1 activation alters the dynamics of pulmonary IRAK$M$ expression in vivo and improves host defense during Pneumococcal pneumonia. J. Immunol. 183, 2027-2036 (2009).

8. Klesney-Tait, J. et al. Transepithelial migration of neutrophils into the lung requires TREM-1. J. Clin. Invest. 123, 138-149 (2013).
9. Gibot, S., Cravoisy, A., Levy, B., Bene, M.-C., Faure, G. \& Bollaert, P.-E. Soluble triggering receptor expressed on myeloid cells and the diagnosis of pneumonia. N. Engl. J. Med. 350, 451-458 (2004).

10. Richeldi, L. et al. Triggering receptor expressed on myeloid cells: role in the diagnosis of lung infections. Eur. Respir. J. 24, 247-250 (2004).

11. Saldir, M. et al. Endocan and soluble triggering receptor expressed on myeloid cells- 1 as novel markers for neonatal sepsis. Pediatr. Neonatol. 56, 415-421 (2015).

12. Arízaga-Ballesteros, V. et al. Can sTREM-1 predict septic shock \& death in late-onset neonatal sepsis? A pilot study. Int. J. Infect. Dis. 30, 27-32 (2015).

13. Su, L. et al. Dynamic changes in serum soluble triggering receptor expressed on myeloid cells-1 (sTREM-1) and its gene polymorphisms are associated with sepsis prognosis. Inflammation 35, 1833-1843 (2012).

14. Stoppelkamp, S., Veseli, K., Stang, K., Schlensak, C., Wendel, H.P. \& Walker, T. Identification of Predictive early biomarkers for sterile-SIRS after cardiovascular surgery. PLOS ONE 10, e0135527 (2015).

15. Zhang, J., She, D., Feng, D., Jia, Y. \& Xie, L. Dynamic changes of serum soluble triggering receptor expressed on myeloid cells-1 (STREM-1) reflect sepsis severity and can predict prognosis: a prospective study. BMC Infect. Dis. 11, 53 (2011).

16. Gibot, S. et al. Time-course of STREM (soluble triggering receptor expressed on myeloid cells)-1, procalcitonin, and C-reactive protein plasma concentrations during sepsis. Crit. Care Med. 33, 792-796 (2005).

17. Smith, A. \& McCullers, J. Secondary bacterial infections in influenza virus infection pathogenesis. In: Influenza Pathogenesis and Control Compans (eds Richard, W. \& Oldstone, M.B.A.) Vol. I, 327-356 (Springer International Publishing: Cham, Switzerland, 2014).

18. Metzger, D.W. \& Sun, K. Immune dysfunction and bacterial coinfections following Influenza. J. Immunol. 191, 2047-2052 (2013).

19. Gomez-Pina, V. et al. Metalloproteinases shed TREM-1 ectodomain from lipopolysaccharide-stimulated human monocytes. J. Immunol. 179, 4065-4073 (2007).

20. Garton, K.J., Gough, P.J. \& Raines, E.W. Emerging roles for ectodomain shedding in the regulation of inflammatory responses. J. Leukoc. Biol. 79, 1105-1116 (2006).

21. Smalley, D.M. \& Ley, K. L-selectin: mechanisms and physiological significance of ectodomain cleavage. J. Cell Mol. Med. 9, 255-266 (2005).

22. Koenen, R.R. et al. Regulated release and functional modulation of junctional adhesion molecule A by disintegrin metalloproteinases. Blood 113, 4799-4809 (2009).

23. McNamara, P.S., Ritson, P., Selby, A., Hart, C.A. \& Smyth, R.L. Bronchoalveolar lavage cellularity in infants with severe respiratory syncytial virus bronchiolitis. Arch. Dis. Child 88, 922-926 (2003).

24. Khokha, R., Murthy, A. \& Weiss, A. Metalloproteinases and their natural inhibitors in inflammation and immunity. Nat. Rev. Immunol. 13, 649-665 (2013).

25. Marcy, A.I. et al. Human fibroblast stromelysin catalytic domain: expression, purification, and characterization of a C-terminally truncated form. Biochemistry 30, 6476-6483 (1991).

26. Vissers, M., Hartman, Y., Groh, L., de Jong, D.J., de Jonge, M.I. \& Ferwerda, G. Recognition of Streptococcus pneumoniae and muramyl dipeptide by NOD2 results in potent induction of MMP-9, which can be controlled by lipopolysaccharide stimulation. Infect. Immun. 82, 49524958 (2014).

27. Renckens, R. et al. Matrix metalloproteinase-9 deficiency impairs host defense against abdominal sepsis. J. Immunol. 176, 3735-3741 (2006).

28. Lee, J., Cacalano, G., Camerato, T., Toy, K., Moore, M.W. \& Wood, W.I. Chemokine binding and activities mediated by the mouse II-8 receptor. J. Immunol. 155, 2158-2164 (1995).

29. Ruiz-GonzÁLez, A. et al. Triggering receptor (TREM-1) expressed on myeloid cells predicts bacteremia better than clinical variables in community-acquired pneumonia. Respirology 16, 321-325 (2011).

30. Gingras, M.-C., Lapillonne, H. \& Margolin, J.F. TREM-1, MDL-1, and DAP12 expression is associated with a mature stage of myeloid development. Mol. Immunol. 38, 817-824 (2002).

31. Hartmann, M., Herrlich, A. \& Herrlich, P. Who decides when to cleave an ectodomain? Trends Biochem. Sci. 38, 111-120 (2013). 
32. Clark, P. Protease-mediated ectodomain shedding. Thorax 69 682-684 (2014).

33. Molad, Y., Pokroy-Shapira, E. \& Carmon, V. CpG-oligodeoxynucleotideinduced TLR9 activation regulates macrophage TREM-1 expression and shedding. Innate Immun. 19, 623-630 (2013).

34. Bratcher, P.E., Weathington, N.M., Nick, H.J., Jackson, P.L., Snelgrove, R.J. \& Gaggar, A. MMP-9 cleaves SP-D and abrogates its innate immune functions in vitro. PLOS ONE 7, e41881 (2012).

35. Yang, C. et al. TREM-1 signaling promotes host defense during the early stage of infection with highly pathogenic Streptococcus suis. Infect. Immun. 83, 3293-3301 (2015).

36. Bouchon, A., Facchetti, F., Weigand, M.A. \& Colonna, M. TREM-1 amplifies inflammation and is a crucial mediator of septic shock. Nature 410, 1103-1107 (2001).

37. Gibot, S. et al. TREM-1 promotes survival during septic shock in mice. Eur J. Immunol. 37, 456-466 (2007).

38. Gibot, S. et al. Modulation of the triggering receptor expressed on the myeloid cell type 1 pathway in murine septic shock. Infect. Immun. 74, 2823-2830 (2006).

39. Gibot, S. et al. Surface and soluble triggering receptor expressed on myeloid cells-1: Expression patterns in murine sepsis. Crit. Care Med. 33, 1787-1793 (2005).

40. Cohen, J. TREM-1 in sepsis. Lancet 358, 776-778 (2001).

41. Nathan, C. \& Ding, A. TREM-1: a new regulator of innate immunity in sepsis syndrome. Nat Med 7, 530-532 (2001).

42. Wang, F. et al. Blocking TREM-1 signaling prolongs survival of mice with Pseudomonas aeruginosa induced sepsis. Cell Immunol 272, 251-258 (2012).

43. Weber, B. et al. TREM-1 deficiency can attenuate disease severity without affecting pathogen clearance. PLoS Pathog. 10, e1003900 (2014).

44. Hommes, T.J. et al. TREM-1/3 contribute to protective Immunity in klebsiella derived pneumosepsis whereas TREM-2 does not. Am. J. Respir. Cell Mol. Biol. 53, 647-655 (2015).

45. Hommes, T.J. et al. Triggering receptor expressed on myeloid cells-1 (TREM-1) improves host defence in pneumococcal pneumonia. J Pathol 233, 357-367 (2014).
46. Kong, M.Y. et al. Pulmonary matrix metalloproteinase-9 activity in mechanically ventilated children with respiratory syncytial virus. Eur. Respir. J. 43, 1086-1096 (2014).

47. Song, J., Wu, C., Zhang, X. \& Sorokin, L.M. In vivo processing of CXCL5 (LIX) by matrix metalloproteinase (MMP)-2 and MMP-9 promotes early neutrophil recruitment in IL-1 $\beta$-induced peritonitis. J. Immunol. 190, 401410 (2013).

48. Van den Steen, P.E., Proost, P., Wuyts, A., Van Damme, J. \& Opdenakker, G. Neutrophil gelatinase B potentiates interleukin-8 tenfold by aminoterminal processing, whereas it degrades CTAP-III, PF-4, and GRO-alpha and leaves RANTES and MCP-2 intact. Blood 96, 2673-2681 (2000).

49. Bradley, L.M., Douglass, M.F., Chatterjee, D., Akira, S. \& Baaten, B.J.G. Matrix metalloprotease 9 mediates neutrophil migration into the airways in response to influenza virus-induced Toll-like receptor signaling. PLoS Pathog. 8, e1002641 (2012).

50. Peng, L.-S., Li, J., Zhou, G.-S., Deng, L.-H. \& Yao, H.-G. Relationships between genetic polymorphisms of triggering receptor expressed on myeloid cells-1 and septic shock in a Chinese Han population. World J. Emerg. Med. 6, 123-130 (2015).

51. Gibot, S. et al. A soluble form of the triggering receptor expressed on myeloid cells-1 modulates the inflammatory response in murine sepsis. J. Exp. Med. 200, 1419-1426 (2004).

(c) (1) () $\fallingdotseq$ This work is licensed under a Creative Commons Ay Attribution-NonCommercial-NoDerivs 4.0 International License. The images or other third party material in this article are included in the article's Creative Commons license, unless indicated otherwise in the credit line; if the material is not included under the Creative Commons license, users will need to obtain permission from the license holder to reproduce the material. To view a copy of this license, visit http://creativecommons.org/licenses/by-nc-nd/4.0/

(C) The Author(s) 2017 